
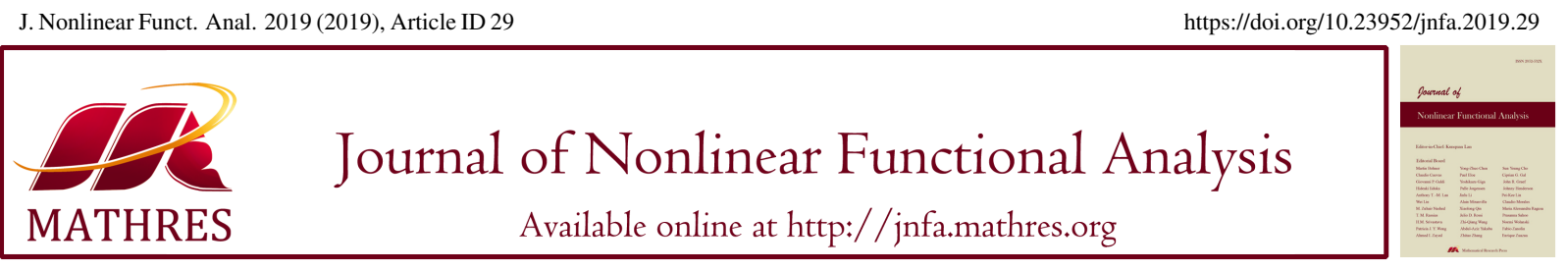

\title{
A SELF-ADAPTIVE ARMIJO-LIKE STEP SIZE METHOD FOR SOLVING MONOTONE VARIATIONAL INEQUALITY PROBLEMS IN HILBERT SPACES
}

\author{
MING TIAN*, MENGYING TONG \\ Tianjin Key Lab for Advanced Signal Processing, Civil Aviation University of China, Tianjin 300300, China
}

\begin{abstract}
In this paper, we study the weak and the strong convergence of a self-adaptive Armijo-like step size method for solving variational inequality problems with monotone and Lipschitz continuous mappings in a real Hilbert space. We combine Tseng's extragradient method with relaxation algorithm and Yamada's algorithm, respectively. It is worth emphasizing that our algorithm do not need to know the Lipschitz constant of the Lipschitz continuous mapping. We also give numerical examples to illustrate our main results.
\end{abstract}

Keywords. Extragradient method; Tseng's extragradient method; Yamada algorithm; Relaxation algorithm; Self-adaptive Armijo-like step size method.

2010 Mathematics Subject Classification. 47H09, 65J15, 90C30.

\section{INTRODUCTION}

Throughout this paper, let $H$ be a real Hilbert space with inner product $\langle\cdot, \cdot\rangle$ and norm $\|\cdot\|$. Let $C$ be a nonempty, closed and convex subset of $H$. Let $\mathbb{N}$ and $\mathbb{R}$ be the sets of positive integers and real numbers, respectively.

Recall that the classical variational inequality problem (VIP) is to find some $x^{*} \in C$ such that

$$
\left\langle A x^{*}, x-x^{*}\right\rangle \geq 0, \quad \forall x \in C .
$$

The set of solutions of problem (1.1) is denoted by $\operatorname{VI}(C, A)$. The variational inequality problem is an important branch of nonlinear problems and it has received a lot of attentions by many authors; see, for instance, $[1,2,3,4,5,6,7]$. There are several ways to solve the variational inequality problem. Under appropriate conditions, there are two general approaches, one is the regularized method and the other is the projection method. In the following, we mainly study the projection method. In particular, we can turn the variational inequality problem into a fixed point problem, in other words, the variational inequality is equivalent to

$$
x^{*}=P_{C}(I-\lambda A) x^{*}
$$

${ }^{*}$ Corresponding author.

E-mail addresses: tianming1963@126.com (M. Tian), tmysugar@163.com (M. Tong).

Received December 5, 2018; Accepted July 1, 2019.

(C)2019 Journal of Nonlinear Functional Analysis 
where $P_{C}: H \rightarrow C$ is the metric projection and $\lambda>0$. The iteration formula

$$
x_{n+1}=P_{C}(I-\lambda A) x_{n},
$$

where $A: H \rightarrow H$ is $\eta$-strongly monotone, $L$-Lipschitz continuous and $\lambda \in\left(0,2 \eta / L^{2}\right)$. It is an extension of the projection gradient method. However, this method requires a slightly assumption that the operator is strongly monotone or inverse strongly monotone.

In 1976, Korpelevich [8] proposed an algorithm which was called as the extragradient method:

$$
\left\{\begin{array}{l}
y_{n}=P_{C}\left(x_{n}-\lambda A x_{n}\right), \\
x_{n+1}=P_{C}\left(x_{n}-\lambda A y_{n}\right)
\end{array}\right.
$$

for each $n=1,2, \ldots$, where $\lambda \in(0,1 / L)$. In particular, $A$ is monotone and $L$-Lipschitz continuous, which avoids the strong assumption for algorithm (1.4). From algorithm (1.4), we clearly know that the extragradient method needs to calculate two projections from $H$ onto the closed convex set $C$ which has increased the difficulty of calculation. Algorithms (1.3) and (1.4) are applicable to the case that $P_{C}$ has a closed form, in other words, $P_{C}$ has an explicit expression. But in some cases, the projection onto the nonempty closed convex subset $C$ might be hard to be implemented. To overcome this difficulty, it has received great attentions by many authors who had improved it in various ways.

To our knowledge, there are four kinds of methods. The first one is the modification of the extragradient method by Tseng [9] who proposed it in 2000 with the following remarkable scheme:

$$
\left\{\begin{array}{l}
y_{n}=P_{C}\left(x_{n}-\lambda A x_{n}\right), \\
x_{n+1}=y_{n}-\lambda\left(A y_{n}-A x_{n}\right),
\end{array}\right.
$$

where $A$ is monotone, $L$-Lipschitz continuous and $\lambda \in(0,1 / L)$. We can easily see that the Tseng's extragradient method only needs to calculate one projection, which is simpler than the extragradient method. The second one is the subgradient extragradient method which was proposed by Censor, Gibali and Reich [10] in 2011:

$$
\left\{\begin{array}{l}
y_{n}=P_{C}\left(x_{n}-\lambda A x_{n}\right), \\
T_{n}=\left\{w \in H \mid\left\langle x_{n}-\lambda A x_{n}-y_{n}, w-y_{n}\right\rangle \leq 0\right\} \\
x_{n+1}=P_{T_{n}}\left(x_{n}-\lambda A y_{n}\right),
\end{array}\right.
$$

where $A$ is monotone, $L$-Lipschitz continuous and $\lambda \in(0,1 / L)$. The key operation of the subgradient extragradient method replaces the second projection onto $C$ of the extragradient method by a projection onto a special constructible half-space. This significantly reduces the difficulty of calculations. The third one is the inertial projection and contraction method which was studied by Dong, Cho and Zhang [11] in 2017:

$$
\left\{\begin{array}{l}
w_{n}=x_{n}+\alpha_{n}\left(x_{n}-x_{n-1}\right), \\
y_{n}=P_{C}\left(w_{n}-\lambda A w_{n}\right), \\
d\left(w_{n}, y_{n}\right)=\left(w_{n}-y_{n}\right)-\lambda\left(A w_{n}-A y_{n}\right), \\
x_{n+1}=w_{n}-\gamma \beta_{n} d\left(w_{n}, y_{n}\right) .
\end{array}\right.
$$

for each $k \geq 1$, where $\gamma \in(0,2), \lambda>0$,

$$
\begin{gathered}
\beta_{n}:=\left\{\begin{array}{l}
\varphi\left(w_{n}, y_{n}\right) /\left\|d\left(w_{n}, y_{n}\right)\right\|^{2}, \quad \text { if } \quad d\left(w_{n}, y_{n}\right) \neq 0, \\
0, \quad \text { if } d\left(w_{n}, y_{n}\right)=0,
\end{array}\right. \\
\varphi\left(w_{n}, y_{n}\right)=\left\langle w_{n}-y_{n}, d\left(w_{n}, y_{n}\right)\right\rangle .
\end{gathered}
$$


The forth one is a self-adaptive algorithm which was called Tseng's extragradient method. It was proposed by Thong and Hieu [12] in 2017. The algorithm is described as follows:

Algorithm 1.1. Step 1: Choose $x_{0} \in H, \gamma>0, l \in(0,1), \mu \in(0,1)$. Step 2: Given the current iterate $x_{n}$, compute

$$
y_{n}=P_{C}\left(x_{n}-\lambda_{n} A x_{n}\right),
$$

where $\lambda_{n}$ is chosen to be the largest $\lambda \in\left\{\gamma, \gamma l, \gamma l^{2}, \cdots\right\}$ satisfying

$$
\lambda\left\|A x_{n}-A y_{n}\right\| \leq \mu\left\|x_{n}-y_{n}\right\| .
$$

If $y_{n}=x_{n}$, then stop and $x_{n}$ is the solution of the variational inequality problem.

Otherwise,

Step 3: Compute the new iterate $x_{n+1}$ via the following iterate formula:

$$
x_{n+1}=y_{n}-\lambda_{n}\left(A y_{n}-A x_{n}\right) .
$$

Set $n:=n+1$ and return to Step 2 .

It is worth mentioning that the Algorithm 1.1 does not require to know the Lipschitz constant of operator A. This is the advantage of Algorithm 1.1 compared with the other three algorithms. Under appropriate conditions, the sequences $\left\{x_{n}\right\}$ generated by (1.5), (1.6), (1.7) and Algorithm 1.1 all converge weakly to an element of $V I(C, A)$. By comparing the above four methods, we find that the conditions of the operator were weaken, but a projection was still preserved during the calculation. So is there a way to avoid the calculation of projections and can it also solve the variational inequality problem?

In 2011, Yamada [13] introduce an algorithmic solution to solve the variational inequality problem which was named the hybrid steepest descent method:

$$
x_{n+1}=\left(I-\mu \alpha_{n} F\right) T x_{n}, \quad \forall n \in \mathbb{N},
$$

where $F: H \rightarrow H$ is $k$-Lipschitz continuous, $\eta$-strongly monotone and $T$ is a nonexpansive mapping. It does not require to calculate $P_{C}$ but requires a closed form expression of a nonexpansive mapping $T$. Assume $\operatorname{Fix}(T) \neq \emptyset$, the sequence $\left\{x_{n}\right\}$ generated by (1.8) converges strongly to a point $x^{*}$, which is a unique solution of the hierarchical variational inequality $\left\langle F x^{*}, x-x^{*}\right\rangle \geq 0, \quad \forall x \in F i x(T)$.

In this paper, motivated and inspired by the above result, we present two new algorithms, which are based on the Tseng's extragradient method. The operator $A$ is monotone and Lipschitz continuous. In particular, the Lipschitz constant of the operator $A$ does not require to be known. We combine the Tseng's extragradient method with the relaxation algorithm and the Yamada's algorithm, respectively. Weak and strong convergence theorems are obtained in Section 3, respectively. This paper is organized as follows. In Section 2, we list some definitions and lemmas which will be used in Section 3. In Section 3, we proposed two new algorithms. Weak and strong convergence theorems are established. In Section 4, we give numerical examples to illustrate the main results. 


\section{PRELIMINARIES}

In this section, we introduce some definitions and lemmas which will be used in this paper.

Assume that $H$ is a real Hilbert space. Let $C$ be a nonempty closed convex subset of $H$. We use the sign $^{\prime} \rightarrow$ to denote that the sequence $\left\{x_{n}\right\}$ converges strongly to a point $x$, i.e., $\left\{x_{n}\right\} \rightarrow x$ and use ${ }^{\prime} \rightarrow^{\prime}$ to denote that the sequence $\left\{x_{n}\right\}$ converges weakly to a point $x$, i.e., $\left\{x_{n}\right\} \rightarrow x$. If there exists a subsequence $\left\{x_{n_{i}}\right\}$ of $\left\{x_{n}\right\}$ converging weakly to a point $z$, then $z$ is called a weak cluster point of $\left\{x_{n}\right\}$. We use $\omega_{w}\left(x_{n}\right)$ to denote the set of all weak cluster points of $\left\{x_{n}\right\}$.

For each $x \in H$, there exists a unique nearest point in $C$, which is denoted by $P_{C} x$ and it satisfies the following property,

$$
\left\|x-P_{C} x\right\|=\inf \{\|x-y\|: y \in C\},
$$

where $P_{C} x: H \rightarrow C$ is called the metric projection from $H$ to $C$. In the following, we gather some useful properties of projections in the Lemma 2.1.

Lemma 2.1. [14] Let $H$ be a real Hilbert space and let $C$ be a nonempty closed subset of $H$. Given $z \in C$, we have:

(i) $\left\|P_{C} x-P_{C} y\right\|^{2} \leq\left\langle x-y, P_{C} x-P_{C} y\right\rangle, \quad \forall x, y \in H$;

(ii) $\left\|x-P_{C} x\right\|^{2}+\left\|y-P_{C} y\right\|^{2} \leq\|x-y\|^{2}, \quad \forall x \in H, y \in C$;

(iii) $z=P_{C} x$ iff $\langle x-z, y-z\rangle \leq 0, \quad \forall x \in H, y \in C$.

From the above properties of projections, we know that $P_{C} x$ is firmly nonexpansive or 1-inverse strongly monotone.

Next, we present some well known results which will be used in the proof of our main results.

Lemma 2.2. [15] Let $\lambda \in \mathbb{R}, \forall x, y \in H$. Then

(i) $\|x+y\|^{2}=\|x\|^{2}+\|y\|^{2}+2\langle x, y\rangle$;

(ii) $\|x+y\|^{2} \leq\|x\|^{2}+2\langle y, x+y\rangle$;

(iii) $\|\lambda x+(1-\lambda) y\|^{2}=\lambda\|x\|^{2}+(1-\lambda)\|y\|^{2}-\lambda(1-\lambda)\|x-y\|^{2}$.

Lemma 2.3. A mapping $A: H \rightarrow H$ is said to be:

(i) contraction iff

$$
\|A x-A y\| \leq \alpha\|x-y\|, \forall x, y \in H,
$$

where $\alpha$ is a real constant in $(0,1)$;

(ii) L-Lipschitizian iff

$$
\|A x-A y\| \leq L\|x-y\|, \forall x, y \in H
$$

where $L$ is a real positive constant;

(iii) monotone iff

$$
\langle x-y, A x-A y\rangle \geq 0, \forall x, y \in H
$$

(iv) strongly monotone iff

$$
\langle x-y, A x-A y\rangle \geq \eta\|x-y\|^{2}, \forall x, y \in H,
$$

where $\eta$ is a real positive constant. In this case, $A$ is said to be $\eta$-strongly monotone.

Lemma 2.4. [16] If $A: C \rightarrow H$ is a continuous and monotone mapping, then $x^{*}$ is a solution of VIP if and only if $x^{*}$ is a solution of the following problem 


$$
\text { find } x \in C \text { such that }\langle A y, y-x\rangle \geq 0, \forall y \in C \text {. }
$$

Lemma 2.5. [17] Assume that $\left\{a_{n}\right\}_{n=0}^{\infty}$ is a nonnegative real sequence such that

$$
a_{n+1} \leq\left(1-\alpha_{n}\right) a_{n}+\alpha_{n} b_{n}, n \geq 0,
$$

where $\left\{\alpha_{n}\right\}$ is a sequence in $(0,1)$ and $\left\{b_{n}\right\}$ is a sequence in $\mathbb{R}$ such that

(i) $\sum_{n=0}^{\infty} \alpha_{n}=\infty$;

(ii) $\lim \sup _{n \rightarrow \infty} b_{n} \leq 0$.

Then $\lim _{n \rightarrow \infty} a_{n}=0$.

Lemma 2.6. [18] Let $C$ be a nonempty closed and convex subset of a real Hilbert space $H$ and let $\left\{x_{n}\right\}$ be a sequence in $H$. The following two properties hold:

(i) $\lim _{n \rightarrow \infty}\left\|x_{n}-x\right\|$ exists for each $x \in C$;

(ii) $\omega_{w}\left(x_{n}\right) \subset C$.

Then the sequence $\left\{x_{n}\right\}$ converges weakly to a point in $C$.

Lemma 2.7. [19] Let $\left\{a_{n}\right\}$ be a sequence of nonnegative real numbers such that there exists a subsequence $\left\{a_{n_{j}}\right\}$ of $\left\{a_{n}\right\}$ such that $a_{n_{j}}<a_{n_{j}+1}$ for all $j \in \mathbb{N}$. Then there exists a nondecreasing sequence $\left\{m_{k}\right\}$ of $\mathbb{N}$ such that $\lim _{k \rightarrow \infty} m_{k}=\infty$ and the following properties are satisfied by all (sufficiently large) number $k \in \mathbb{N}: a_{m_{k}} \leq a_{m_{k}+1}$ and $a_{k} \leq a_{m_{k}+1}$. In fact, $m_{k}$ is the largest number of $n$ in the set $\{1,2, \cdots, k\}$ such that $a_{n}<a_{n+1}$.

\section{MAIN RESULTS}

In this section, we propose two new iterative algorithms with a self-adaptive Armijo-like step size method. We combine Tseng's extragradient method with relaxation algorithm and Yamada's algorithm, respectively. They are mainly used to solve the monotone and Lipschitz continuous variational inequality problem without the knowledge of the operator $A: H \rightarrow H$. In particular, under the assumption $\operatorname{VI}(C, A) \neq \emptyset$, we prove a strong convergence theorem which is suitable for solving the hierarchical variational inequality problems.

3.1. The weak convergence theorem. In this subsection, we propose a weakly convergent algorithm for monotone variational inequalities. It is combined the Tseng's extragradient method with the relaxation algorithm. Let $A: H \rightarrow H$ be a monotone and Lipschitz continuous operator. Assume $V I(C, A) \neq \emptyset$ and our algorithm is described as follows.

Algorithm 3.1. Step 1: Choose $x_{0} \in H, \gamma>0, l \in(0,1), \mu \in(0,1), \alpha_{n} \in[a, b],[a, b] \subset(0,1)$.

Step 2: Given the current iterate $x_{n}$, compute

$$
y_{n}=P_{C}\left(x_{n}-\lambda_{n} A x_{n}\right),
$$

where $\lambda_{n}$ is chosen to be the largest $\lambda \in\left\{\gamma, \gamma l, \gamma l^{2}, \cdots\right\}$ satisfying

$$
\lambda\left\|A x_{n}-A y_{n}\right\| \leq \mu\left\|x_{n}-y_{n}\right\| .
$$

If $y_{n}=x_{n}$, then stop and $x_{n}$ is the solution of the variational inequality problem. 
Otherwise,

Step 3: Compute the new iterate $x_{n+1}$ via the following iterate formula:

$$
x_{n+1}=\left(1-\alpha_{n}\right) x_{n}+\alpha_{n} z_{n},
$$

where $z_{n}=y_{n}-\lambda_{n}\left(A y_{n}-A x_{n}\right)$.

Set $n:=n+1$ and return to Step 2 .

Lemma 3.2. The sequence $\left\{\lambda_{n}\right\}$ generated by Algorithm 3.1 is well defined and

$$
\min \left\{\gamma, \frac{\mu l}{L}\right\} \leq \lambda_{n} \leq \gamma
$$

Proof. It is obvious that the sequence $\left\{\lambda_{n}\right\}$ is well defined. In fact, since $A$ is $L$-Lipschitz continuous, we have

$$
\left\|A x_{n}-A\left(P_{C}\left(x_{n}-\lambda A x_{n}\right)\right)\right\| \leq L\left\|x_{n}-P_{C}\left(x_{n}-\lambda A x_{n}\right)\right\|,
$$

which can be transformed into

$$
\frac{\mu}{L}\left\|A x_{n}-A\left(P_{C}\left(x_{n}-\lambda A x_{n}\right)\right)\right\| \leq \mu\left\|x_{n}-P_{C}\left(x_{n}-\lambda A x_{n}\right)\right\| .
$$

This implies that, for all $\lambda \leq \frac{\mu}{L}$, formula (3.1) is established. So $\lambda_{n}$ is well defined. Because $\lambda_{n}$ is chosen to be the largest $\lambda \in\left\{\gamma, \gamma l, \gamma l^{2}, \ldots\right\}$ satisfying

$$
\lambda\left\|A x_{n}-A y_{n}\right\| \leq \mu\left\|x_{n}-y_{n}\right\|,
$$

where $\gamma>0, l \in(0,1)$, we have $\lambda_{n} \leq \gamma$. If $\lambda_{n}=\gamma$, then the lemma is proved. If $\lambda_{n}<\gamma$, since for all $\lambda \leq \frac{\mu}{L}$, formula (3.1) is established, $\lambda_{n}=\gamma l^{p}(p>1)$, then $\lambda_{n} l^{-1}>\frac{\mu}{L}$. Hence, we obtain $\lambda_{n}>\frac{\mu l}{L}$. Clearly,

$$
\min \left\{\gamma, \frac{\mu l}{L}\right\} \leq \lambda_{n} \leq \gamma
$$

Lemma 3.3. Assume that the sequences $\left\{x_{n}\right\},\left\{y_{n}\right\},\left\{z_{n}\right\}$ generated by Algorithm 3.1 and $\lim _{n \rightarrow \infty} \| x_{n}-$ $y_{n} \|=0$. If $\left\{x_{n_{k}}\right\}$ converges weakly to some $z \in H$, then $z \in V I(C, A)$.

Proof. Since $A$ is monotone and $y_{n_{k}}=P_{C}\left(x_{n_{k}}-\lambda_{n_{k}} A x_{n_{k}}\right)$, by Lemma 2.1 (iii), we have

$$
\left\langle y_{n_{k}}-x_{n_{k}}+\lambda_{n_{k}} A x_{n_{k}}, x-y_{n_{k}}\right\rangle \geq 0, \quad \forall x \in C .
$$

Then,

$$
\begin{aligned}
0 \leq & \left\langle y_{n_{k}}-x_{n_{k}}, x-y_{n_{k}}\right\rangle+\lambda_{n_{k}}\left\langle A x_{n_{k}}, x-y_{n_{k}}\right\rangle \\
= & \left\langle y_{n_{k}}-x_{n_{k}}, x-y_{n_{k}}\right\rangle+\lambda_{n_{k}}\left\langle A x_{n_{k}}, x_{n_{k}}-y_{n_{k}}+x-x_{n_{k}}\right\rangle \\
= & \left\langle y_{n_{k}}-x_{n_{k}}, x-y_{n_{k}}\right\rangle+\lambda_{n_{k}}\left\langle A x_{n_{k}}, x_{n_{k}}-y_{n_{k}}\right\rangle \\
& +\lambda_{n_{k}}\left\langle A x_{n_{k}}, x-x_{n_{k}}\right\rangle \\
\leq & \left\langle y_{n_{k}}-x_{n_{k}}, x-y_{n_{k}}\right\rangle+\lambda_{n_{k}}\left\langle A x_{n_{k}}, x_{n_{k}}-y_{n_{k}}\right\rangle \\
& +\lambda_{n_{k}}\left\langle A x, x-x_{n_{k}}\right\rangle, \quad \forall x \in C .
\end{aligned}
$$

By Lemma 3.2, we have $\lambda_{n}>\frac{\mu l}{L}$. Letting $k \rightarrow \infty$ and using $\lim _{k \rightarrow \infty}\left\|x_{n_{k}}-y_{n_{k}}\right\|=0$, we obtain

$$
\langle A x, x-z\rangle \geq 0, \quad \forall x \in C .
$$

Using Lemma 2.4, we have $z \in V I(C, A)$. 
Theorem 3.4. Let $\left\{x_{n}\right\}$ be a sequence generated by Algorithm 3.1. Then $\left\{x_{n}\right\}$ converges weakly to a point of $\operatorname{VI}(C, A)$.

Proof. For any $p \in V I(C, A)$, we have

$$
\begin{aligned}
\left\|x_{n+1}-p\right\|^{2}= & \left\|\left(1-\alpha_{n}\right) x_{n}+\alpha_{n} z_{n}-p\right\|^{2} \\
= & \left\|\left(1-\alpha_{n}\right)\left(x_{n}-p\right)+\alpha_{n}\left(z_{n}-p\right)\right\|^{2} \\
= & \left(1-\alpha_{n}\right)\left\|x_{n}-p\right\|^{2}+\alpha_{n}\left\|z_{n}-p\right\|^{2} \\
& -\alpha_{n}\left(1-\alpha_{n}\right)\left\|x_{n}-z_{n}\right\|^{2} .
\end{aligned}
$$

Since $z_{n}=y_{n}-\lambda_{n}\left(A y_{n}-A x_{n}\right)$, we have

$$
\begin{aligned}
\left\|z_{n}-p\right\|^{2}= & \left\|y_{n}-\lambda_{n}\left(A y_{n}-A x_{n}\right)-p\right\|^{2} \\
= & \left\|y_{n}-p\right\|^{2}+\lambda \lambda_{n}^{2}\left\|A y_{n}-A x_{n}\right\|^{2}-2 \lambda_{n}\left\langle y_{n}-p, A y_{n}-A x_{n}\right\rangle \\
= & \left\|y_{n}-x_{n}+x_{n}-p\right\|^{2}+\lambda_{n}^{2}\left\|A y_{n}-A x_{n}\right\|^{2}-2 \lambda_{n}\left\langle y_{n}-p, A y_{n}-A x_{n}\right\rangle \\
= & \left\|x_{n}-p\right\|^{2}+\left\|x_{n}-y_{n}\right\|^{2}+2\left\langle y_{n}-x_{n}, x_{n}-p\right\rangle+\lambda_{n}^{2}\left\|A y_{n}-A x_{n}\right\|^{2} \\
& -2 \lambda_{n}\left\langle y_{n}-p, A y_{n}-A x_{n}\right\rangle \\
= & \left\|x_{n}-p\right\|^{2}+\left\|x_{n}-y_{n}\right\|^{2}-2\left\langle y_{n}-x_{n}, y_{n}-x_{n}\right\rangle \\
& +2\left\langle y_{n}-x_{n}, y_{n}-p\right\rangle+\lambda_{n}^{2}\left\|A y_{n}-A x_{n}\right\|^{2}-2 \lambda_{n}\left\langle y_{n}-p, A y_{n}-A x_{n}\right\rangle \\
= & \left\|x_{n}-p\right\|^{2}+\left\|x_{n}-y_{n}\right\|^{2}-2\left\|x_{n}-y_{n}\right\|^{2}+2\left\langle y_{n}-x_{n}, y_{n}-p\right\rangle \\
& +\lambda_{n}^{2}\left\|A y_{n}-A x_{n}\right\|^{2}-2 \lambda_{n}\left\langle y_{n}-p, A y_{n}-A x_{n}\right\rangle \\
= & \left\|x_{n}-p\right\|^{2}-\left\|x_{n}-y_{n}\right\|^{2}+2\left\langle y_{n}-x_{n}, y_{n}-p\right\rangle+\lambda_{n}^{2}\left\|A y_{n}-A x_{n}\right\|^{2} \\
& -2 \lambda_{n}\left\langle y_{n}-p, A y_{n}-A x_{n}\right\rangle .
\end{aligned}
$$

Since $y_{n}=P_{C}\left(x_{n}-\lambda_{n} A x_{n}\right)$, we have

$$
\left\langle y_{n}-x_{n}+\lambda_{n} A x_{n}, y_{n}-p\right\rangle \leq 0,
$$

which is equivalent to

$$
\left\langle y_{n}-x_{n}, y_{n}-p\right\rangle \leq-\lambda_{n}\left\langle A x_{n}, y_{n}-p\right\rangle .
$$

Besides, we have

$$
\begin{aligned}
\left\|x_{n}-z_{n}\right\|^{2}= & \left\|x_{n}-y_{n}+\lambda_{n}\left(A y_{n}-A x_{n}\right)\right\|^{2} \\
= & \left\|x_{n}-y_{n}\right\|^{2}+\lambda_{n}^{2}\left\|A y_{n}-A x_{n}\right\|^{2} \\
& +2 \lambda_{n}\left\langle x_{n}-y_{n}, A y_{n}-A x_{n}\right\rangle .
\end{aligned}
$$


From (3.2), (3.3), (3.4) and (3.5), we have

$$
\begin{aligned}
\left\|x_{n+1}-p\right\|^{2}= & \left(1-\alpha_{n}\right)\left\|x_{n}-p\right\|^{2}+\alpha_{n}\left\|x_{n}-p\right\|^{2}-\alpha_{n}\left\|x_{n}-y_{n}\right\|^{2} \\
& +2 \alpha_{n}\left\langle y_{n}-x_{n}, y_{n}-p\right\rangle+\alpha_{n} \lambda_{n}^{2}\left\|A y_{n}-A x_{n}\right\|^{2} \\
& -2 \alpha_{n} \lambda_{n}\left\langle y_{n}-p, A y_{n}-A x_{n}\right\rangle-\alpha_{n}\left(1-\alpha_{n}\right)\left\|x_{n}-y_{n}\right\|^{2} \\
& -\alpha_{n}\left(1-\alpha_{n}\right) \lambda_{n}^{2}\left\|A y_{n}-A x_{n}\right\|^{2} \\
& -2 \alpha_{n}\left(1-\alpha_{n}\right) \lambda_{n}\left\langle x_{n}-y_{n}, A y_{n}-A x_{n}\right\rangle \\
\leq & \left\|x_{n}-p\right\|^{2}+\left(\alpha_{n}^{2}-2 \alpha_{n}\right)\left\|x_{n}-y_{n}\right\|^{2}+\alpha_{n}^{2} \lambda_{n}^{2}\left\|A y_{n}-A x_{n}\right\|^{2} \\
& -2 \alpha_{n} \lambda_{n}\left\langle y_{n}-p, A x_{n}\right\rangle-2 \alpha_{n} \lambda_{n}\left\langle y_{n}-p, A y_{n}-A x_{n}\right\rangle \\
& -2 \alpha_{n}\left(1-\alpha_{n}\right) \lambda_{n}\left\langle x_{n}-y_{n}, A y_{n}-A x_{n}\right\rangle \\
= & \left\|x_{n}-p\right\|^{2}+\left(\alpha_{n}^{2}-2 \alpha_{n}\right)\left\|x_{n}-y_{n}\right\|^{2}+\alpha_{n}^{2} \lambda_{n}^{2}\left\|A y_{n}-A x_{n}\right\|^{2} \\
& -2 \alpha_{n} \lambda_{n}\left\langle y_{n}-p, A y_{n}\right\rangle+2 \alpha_{n}\left(1-\alpha_{n}\right) \lambda_{n}\left\langle x_{n}-y_{n}, A x_{n}-A y_{n}\right\rangle \\
\leq & \left\|x_{n}-p\right\|^{2}+\left(\alpha_{n}^{2}-2 \alpha_{n}\right)\left\|x_{n}-y_{n}\right\|^{2}+\alpha_{n}^{2} \lambda_{n}^{2}\left\|A y_{n}-A x_{n}\right\|^{2} \\
& -2 \alpha_{n} \lambda_{n}\left(\left\langle y_{n}-p, A y_{n}-A p\right\rangle+\left\langle y_{n}-p, A p\right\rangle\right) \\
& +2 \alpha_{n}\left(1-\alpha_{n}\right) \lambda_{n}\left\|x_{n}-y_{n}\right\|\left\|A x_{n}-A y_{n}\right\| \\
\leq & \left\|x_{n}-p\right\|^{2}+\left(\alpha_{n}^{2}-2 \alpha_{n}\right)\left\|x_{n}-y_{n}\right\|^{2}+\alpha_{n}^{2} \mu^{2}\left\|x_{n}-y_{n}\right\|^{2} \\
& +2 \alpha_{n}\left(1-\alpha_{n}\right) \mu\left\|x_{n}-y_{n}\right\|^{2} \\
\leq & \left\|x_{n}-p\right\|^{2}+\left(2 \alpha_{n}^{2}-2 \alpha_{n}+2 \alpha_{n}\left(1-\alpha_{n}\right) \mu\right)\left\|x_{n}-y_{n}\right\|^{2} \\
= & \left\|x_{n}-p\right\|^{2}-2 \alpha_{n}\left(1-\alpha_{n}-\left(1-\alpha_{n}\right) \mu\right)\left\|x_{n}-y_{n}\right\|^{2} \\
\leq & \left\|x_{n}-p\right\|^{2}-2 a(1-b)(1-\mu)\left\|x_{n}-y_{n}\right\|^{2} \\
\leq & \left\|x_{n}-p\right\|^{2}
\end{aligned}
$$

This implies that the sequence $\left\{x_{n}\right\}$ is bounded. So $\lim _{n \rightarrow \infty}\left\|x_{n}-p\right\|$ exists and $\lim _{n \rightarrow \infty}\left\|x_{n}-y_{n}\right\|=0$ for each $p \in V I(C, A)$. Because $\left\{x_{n}\right\}$ is bounded, there exists a subsequence $\left\{x_{n_{k}}\right\}$ of $\left\{x_{n}\right\}$ such that $\left\{x_{n_{k}}\right\}$ converges weakly to $z \in H$. By Lemma 3.3, we obtain that $z \in V I(C, A)$. Therefore, it follows from Lemma 2.6 that $\left\{x_{n}\right\}$ converges weakly to a point of $V I(C, A)$. This is the desired result.

3.2. The strong convergence theorem. In this subsection, we propose a strongly convergent algorithm for hierarchical monotone variational inequalities. It is combined the Tseng's extragradient method with Yamada's algorithm. Let $A: H \rightarrow H$ be a monotone and Lipschitz continuous operator. In particular, we do not require the knowledge of the Lipschitz constant of the mapping $A$. Let $F: H \rightarrow H$ be $\eta$-strongly monotone and $k$-Lipschitz continuous, where $\eta>0$ and $k>0$. Assume $V I(C, A) \neq \emptyset$. Let $\left\{x_{n}\right\}$ be a sequence generated by the following algorithm.

Algorithm 3.5. Step 1: Choose $x_{0} \in H, \gamma>0, l \in(0,1), \mu \in(0,1)$.

Step 2: Given the current iterate $x_{n}$, compute

$$
y_{n}=P_{C}\left(x_{n}-\lambda_{n} A x_{n}\right),
$$

where $\lambda_{n}$ is chosen to be the largest $\lambda \in\left\{\gamma, \gamma l, \gamma l^{2}, \cdots\right\}$ satisfying

$$
\lambda\left\|A x_{n}-A y_{n}\right\| \leq \mu\left\|x_{n}-y_{n}\right\| .
$$


If $y_{n}=x_{n}$, then stop and $x_{n}$ is the solution of the variational inequality problem.

Otherwise,

Step 3: Compute the new iterate $x_{n+1}$ via the following iterate formula:

$$
x_{n+1}=\left(I-\beta \alpha_{n} F\right) z_{n},
$$

where $z_{n}=y_{n}-\lambda_{n}\left(A y_{n}-A x_{n}\right)$.

Set $n:=n+1$ and return to Step 2 .

Theorem 3.6. Assume that the parameter sequences $\left\{\alpha_{n}\right\} \subset(0,1), \lim _{n \rightarrow \infty} \alpha_{n}=0, \Sigma_{n=0}^{\infty} \alpha_{n}=\infty$ and $\beta \in$ $\left(0, \frac{2 \eta}{k^{2}}\right)$. Then the sequence $\left\{x_{n}\right\}$ generated by Algorithm 3.5 converges strongly to $q=P_{V I(C, A)}(I-\beta F) q$.

Proof. Now we show that $I-\beta F$ is a contractive mapping. Since $F: H \rightarrow H$ is $\eta$-strongly monotone and $k$-Lipschitz continuous, we have

$$
\begin{aligned}
\|(I-\beta F) x-(I-\beta F) y\|^{2} & =\|x-y-\beta(F x-F y)\|^{2} \\
& =\|x-y\|^{2}+\beta^{2}\|F x-F y\|^{2}-2 \beta\langle x-y, F x-F y\rangle \\
& \leq\|x-y\|^{2}+\beta^{2} k^{2}\|x-y\|^{2}-2 \beta \eta\|x-y\|^{2} \\
& =\left(1-\beta\left(2 \eta-\beta k^{2}\right)\right)\|x-y\|^{2} \\
& \leq(1-\tau)^{2}\|x-y\|^{2}
\end{aligned}
$$

where $\tau=\frac{1}{2} \beta\left(2 \eta-\beta k^{2}\right)$. So $I-\beta F: H \rightarrow H$ is a contraction mapping with constant $1-\tau$. Thus, the mapping $P_{V I(C, A)}(I-\beta F)$ is a contraction. By Banach contraction principle, there exists a unique point $q \in H$ such that $q=P_{V I(C, A)}(I-\beta F) q$. In particular, $q \in V I(C, A)$, we obtain from Lemma 2.1 (iii) that

$$
\langle(I-\beta F) q-q, z-q\rangle \leq 0, \quad \forall z \in V I(C, A) .
$$

First, we verify that the sequence $\left\{x_{n}\right\}$ is bounded. Letting $p \in V I(C, A)$ by (3.3) and (3.4), we have

$$
\begin{aligned}
\left\|z_{n}-p\right\|^{2} \leq & \left\|x_{n}-p\right\|^{2}-\left\|x_{n}-y_{n}\right\|^{2}-2 \lambda_{n}\left\langle A x_{n}, y_{n}-p\right\rangle \\
& +\lambda_{n}^{2}\left\|A y_{n}-A x_{n}\right\|^{2}-2 \lambda_{n}\left\langle y_{n}-p, A y_{n}-A x_{n}\right\rangle \\
= & \left\|x_{n}-p\right\|^{2}-\left\|x_{n}-y_{n}\right\|^{2}+\lambda_{n}^{2}\left\|A y_{n}-A x_{n}\right\|^{2} \\
& -2 \lambda_{n}\left\langle y_{n}-p, A y_{n}\right\rangle \\
\leq & \left\|x_{n}-p\right\|^{2}-\left\|x_{n}-y_{n}\right\|^{2}+\mu^{2}\left\|x_{n}-y_{n}\right\|^{2} \\
& -2 \lambda_{n}\left\langle y_{n}-p, A y_{n}-A p\right\rangle-2 \lambda_{n}\left\langle y_{n}-p, A p\right\rangle \\
\leq & \left\|x_{n}-p\right\|^{2}-\left(1-\mu^{2}\right)\left\|x_{n}-y_{n}\right\|^{2} \\
\leq & \left\|x_{n}-p\right\|^{2} .
\end{aligned}
$$

It implies that

$$
\left\|z_{n}-p\right\| \leq\left\|x_{n}-p\right\|
$$


In fact,

$$
\begin{aligned}
\left\|\left(I-\beta \alpha_{n} F\right) x-\left(I-\beta \alpha_{n} F\right) y\right\|^{2}= & \left\|(x-y)-\beta \alpha_{n}(F x-F y)\right\|^{2} \\
= & \|x-y\|^{2}+\beta^{2} \alpha_{n}^{2}\|F x-F y\|^{2} \\
& -2 \beta \alpha_{n}\langle x-y, F x-F y\rangle \\
\leq & \|x-y\|^{2}+\beta^{2} \alpha_{n}^{2} k^{2}\|x-y\|^{2}-2 \beta \alpha_{n} \eta\|x-y\|^{2} \\
= & \left(1-\beta \alpha_{n}\left(2 \eta-\beta \alpha_{n} k^{2}\right)\right)\|x-y\|^{2} \\
\leq & \left(1-\tau \alpha_{n}\right)^{2}\|x-y\|^{2},
\end{aligned}
$$

where $\tau=\frac{1}{2} \beta\left(2 \eta-\beta k^{2}\right)$. Thus, the mapping $I-\beta \alpha_{n} F$ is a contraction mapping with constant $1-\tau \alpha_{n}$. Therefore,

$$
\begin{aligned}
\left\|x_{n+1}-p\right\| & =\left\|\left(I-\beta \alpha_{n} F\right) z_{n}-p\right\| \\
& =\left\|\left(I-\beta \alpha_{n} F\right) z_{n}-\left(I-\beta \alpha_{n} F\right) p-\beta \alpha_{n} F p\right\| \\
& \leq\left\|\left(I-\beta \alpha_{n} F\right) z_{n}-\left(I-\beta \alpha_{n} F\right) p\right\|+\beta \alpha_{n}\|F p\| \\
& \leq\left(1-\tau \alpha_{n}\right)\left\|z_{n}-p\right\|+\beta \alpha_{n}\|F p\| \\
& =\left(1-\tau \alpha_{n}\right)\left\|z_{n}-p\right\|+\tau \alpha_{n} \frac{\beta}{\tau}\|F p\| \\
& \leq \max \left\{\left\|x_{n}-p\right\|, \frac{\beta}{\tau}\|F p\|\right\} .
\end{aligned}
$$

Hence,

$$
\left\|x_{n+1}-p\right\| \leq \max \left\{\left\|x_{0}-p\right\|, \frac{\beta}{\tau}\|F p\|\right\} .
$$

This implies that the sequence $\left\{x_{n}\right\}$ is bounded. At the same time, $\left\{y_{n}\right\},\left\{z_{n}\right\}$ and $\left\{(I-\beta F) x_{n}\right\}$ are bounded. Next, we show that

$$
\left(1-\alpha_{n}\right)\left(1-\mu^{2}\right)\left\|x_{n}-y_{n}\right\|^{2} \leq\left\|x_{n}-q\right\|^{2}-\left\|x_{n+1}-q\right\|^{2}+\alpha_{n}\left\|(I-\beta F) z_{n}-q\right\|^{2} .
$$

Indeed,

$$
\begin{aligned}
\left\|x_{n+1}-q\right\|^{2}= & \left\|\left(I-\beta \alpha_{n} F\right) z_{n}-q\right\|^{2} \\
= & \left\|\alpha_{n}(I-\beta F) z_{n}+\left(1-\alpha_{n}\right) z_{n}-q\right\|^{2} \\
= & \left\|\alpha_{n}\left((I-\beta F) z_{n}-q\right)+\left(1-\alpha_{n}\right)\left(z_{n}-q\right)\right\|^{2} \\
= & \alpha_{n}\left\|(I-\beta F) z_{n}-q\right\|^{2}+\left(1-\alpha_{n}\right)\left\|z_{n}-q\right\|^{2} \\
& -\alpha_{n}\left(1-\alpha_{n}\right)\left\|\beta F z_{n}\right\|^{2} \\
\leq & \alpha_{n}\left\|(I-\beta F) z_{n}-q\right\|^{2}+\left(1-\alpha_{n}\right)\left\|x_{n}-q\right\|^{2} \\
& -\left(1-\alpha_{n}\right)\left(1-\mu^{2}\right)\left\|x_{n}-y_{n}\right\|^{2} \\
\leq & \alpha_{n}\left\|(I-\beta F) z_{n}-q\right\|^{2}+\left\|x_{n}-q\right\|^{2} \\
& -\left(1-\alpha_{n}\right)\left(1-\mu^{2}\right)\left\|x_{n}-y_{n}\right\|^{2} .
\end{aligned}
$$

This implies that

$$
\left(1-\alpha_{n}\right)\left(1-\mu^{2}\right)\left\|x_{n}-y_{n}\right\|^{2} \leq\left\|x_{n}-q\right\|^{2}-\left\|x_{n+1}-q\right\|^{2}+\alpha_{n}\left\|(I-\beta F) z_{n}-q\right\|^{2} .
$$


Finally, we verify that the sequence $\left\{x_{n}\right\}$ converges strongly to $q$. In the following, we consider the sequence $\left\{\left\|x_{n}-q\right\|\right\}$ from two possible cases.

Case 1. Suppose that there exists $N \in \mathbb{N}$, such that

$$
\left\|x_{n+1}-q\right\| \leq\left\|x_{n}-q\right\|, \quad \forall n>N .
$$

Then, $\lim _{n \rightarrow \infty}\left\|x_{n}-q\right\|$ exists. From (3.14), and $\lim _{n \rightarrow \infty}\left(1-\alpha_{n}\right)\left(1-\mu^{2}\right)=1-\mu^{2}>0, \lim _{n \rightarrow \infty} \alpha_{n}=0$, we can obtain that

$$
\lim _{n \rightarrow \infty}\left\|x_{n}-y_{n}\right\|=0
$$

Since $\left\{x_{n}\right\}$ is bounded, there exists a subsequence $\left\{x_{n_{k}}\right\}$ of $\left\{x_{n}\right\}$ converges weakly to some $z \in H$ such that

$$
\begin{aligned}
\limsup _{n \rightarrow \infty}\left\langle(I-\beta F) q-q, x_{n}-q\right\rangle & =\lim _{k \rightarrow \infty}\left\langle(I-\beta F) q-q, x_{n_{k}}-q\right\rangle \\
& =\langle(I-\beta F) q-q, z-q\rangle .
\end{aligned}
$$

Combining Lemma 3.3 and (3.15), we have $z \in V I(C, A)$. It follows from Lemma 2.1 (iii) that

$$
\limsup _{n \rightarrow \infty}\left\langle(I-\beta F) q-q, x_{n}-q\right\rangle=\langle(I-\beta F) q-q, z-q\rangle \leq 0 .
$$

Thus,

$$
\langle F q, z-q\rangle \geq 0, \quad z \in V I(C, A) .
$$

On the other hand,

$$
\begin{aligned}
\left\|z_{n}-y_{n}\right\| & =\left\|y_{n}-\lambda_{n}\left(A y_{n}-A x_{n}\right)-y_{n}\right\| \\
& =\lambda_{n}\left\|A y_{n}-A x_{n}\right\| \\
& \leq \mu\left\|y_{n}-x_{n}\right\| \rightarrow 0, \quad(n \rightarrow \infty) .
\end{aligned}
$$

Therefore,

$$
\begin{aligned}
\left\|x_{n+1}-x_{n}\right\| & =\left\|\left(I-\beta \alpha_{n} F\right) z_{n}-x_{n}\right\| \\
& =\left\|\alpha_{n}(I-\beta F) z_{n}+\left(1-\alpha_{n}\right) z_{n}-x_{n}\right\| \\
& \leq \alpha_{n}\left\|(I-\beta F) z_{n}-x_{n}\right\|+\left(1-\alpha_{n}\right)\left\|z_{n}-x_{n}\right\| \\
& \leq \alpha_{n}\left\|(I-\beta F) z_{n}-x_{n}\right\|+\left\|z_{n}-x_{n}\right\| \\
& \leq \alpha_{n}\left\|(I-\beta F) z_{n}-x_{n}\right\|+\left\|z_{n}-y_{n}\right\|+\left\|y_{n}-x_{n}\right\| .
\end{aligned}
$$

We obtain that $\lim _{n \rightarrow \infty}\left\|x_{n+1}-x_{n}\right\|=0$. It implies that

$$
\begin{aligned}
\limsup _{n \rightarrow \infty}\left\langle(I-\beta F) q-q, x_{n+1}-q\right\rangle= & \lim _{n \rightarrow \infty}\left\langle(I-\beta F) q-q, x_{n+1}-x_{n}\right\rangle \\
& +\limsup _{n \rightarrow \infty}\left\langle(I-\beta F) q-q, x_{n}-q\right\rangle \\
\leq & 0 .
\end{aligned}
$$


Indeed,

$$
\begin{aligned}
\left\|x_{n+1}-q\right\|^{2}= & \left\|\left(I-\beta \alpha_{n} F\right) z_{n}-q\right\|^{2} \\
= & \left\|\alpha_{n}(I-\beta F) z_{n}+\left(1-\alpha_{n}\right) z_{n}-q\right\|^{2} \\
= & \left\|\alpha_{n}\left((I-\beta F) z_{n}-q\right)+\left(1-\alpha_{n}\right)\left(z_{n}-q\right)\right\|^{2} \\
\leq & \left(1-\alpha_{n}\right)^{2}\left\|z_{n}-q\right\|^{2}+2 \alpha_{n}\left\langle(I-\beta F) z_{n}-q, x_{n+1}-q\right\rangle \\
= & \left(1-\alpha_{n}\right)^{2}\left\|z_{n}-q\right\|^{2}+2 \alpha_{n}\left\langle(I-\beta F) z_{n}-(I-\beta F) q\right. \\
& \left.+(I-\beta F) q-q, x_{n+1}-q\right\rangle \\
= & \left(1-\alpha_{n}\right)^{2}\left\|z_{n}-q\right\|^{2}+2 \alpha_{n}\left\langle(I-\beta F) z_{n}-(I-\beta F) q, x_{n+1}-q\right\rangle \\
& +2 \alpha_{n}\left\langle(I-\beta F) q-q, x_{n+1}-q\right\rangle \\
\leq & \left(1-\alpha_{n}\right)^{2}\left\|z_{n}-q\right\|^{2}+2 \alpha_{n}(1-\tau)\left\|z_{n}-q\right\|\left\|x_{n+1}-q\right\| \\
& +2 \alpha_{n}\left\langle(I-\beta F) q-q, x_{n+1}-q\right\rangle \\
\leq & \left(1-\alpha_{n}\right)^{2}\left\|x_{n}-q\right\|^{2}+2 \alpha_{n}(1-\tau)\left\|x_{n}-q\right\|\left\|x_{n+1}-q\right\| \\
& +2 \alpha_{n}\left\langle(I-\beta F) q-q, x_{n+1}-q\right\rangle \\
\leq & \left(1-2 \alpha_{n}+2 \alpha_{n}(1-\tau)\right)\left\|x_{n}-q\right\|^{2}+\alpha_{n}^{2}\left\|x_{n}-q\right\|^{2} \\
& +2 \alpha_{n}\left\langle(I-\beta F) q-q, x_{n+1}-q\right\rangle \\
= & \left(1-2 \alpha_{n} \tau\right)\left\|x_{n}-q\right\|^{2} \\
& +2 \alpha_{n} \tau\left[\frac{\alpha_{n}\left\|x_{n}-q\right\|^{2}}{2 \tau}+\frac{\left\langle(I-\beta F) q-q, x_{n+1}-q\right\rangle}{\tau}\right] . \\
& (3.18), w e h a v e
\end{aligned}
$$

Combining $\lim _{n \rightarrow \infty} \alpha_{n}=0$ and (3.18), we have

$$
\limsup _{n \rightarrow \infty}\left[\frac{\alpha_{n}\left\|x_{n}-q\right\|^{2}}{2 \tau}+\frac{\left\langle(I-\beta F) q-q, x_{n+1}-q\right\rangle}{\tau}\right] \leq 0 .
$$

Using Lemma 2.5, we get

$$
\lim _{n \rightarrow \infty}\left\|x_{n}-q\right\|=0 \text {. }
$$

This implies that the sequence $\left\{x_{n}\right\}$ converges strongly to $q$.

Case 2. There exists a subsequence $\left\{x_{n_{j}}\right\}$ of $\left\{x_{n}\right\}$ such that $\left\|x_{n_{j}}-q\right\|<\left\|x_{n_{j}+1}-q\right\|$ for all $j \in \mathbb{N}$. From Lemma 2.7, there exists a nondecreasing sequence $\left\{m_{k}\right\}$ of $\mathbb{N}$ such that $\lim _{k \rightarrow \infty} m_{k}=\infty$ and the following inequalities hold for all $k \in \mathbb{N}$ :

$$
\left\|x_{m_{k}}-q\right\| \leq\left\|x_{m_{k}+1}-q\right\| \quad \text { and } \quad\left\|x_{k}-q\right\| \leq\left\|x_{m_{k}+1}-q\right\| .
$$

By (3.14), we obtain

$$
\begin{aligned}
\left(1-\alpha_{m_{k}}\right)\left(1-\mu^{2}\right)\left\|x_{m_{k}}-y_{m_{k}}\right\|^{2} \leq & \left\|x_{m_{k}}-q\right\|^{2}-\left\|x_{m_{k}+1}-q\right\|^{2} \\
& +\alpha_{m_{k}}\left\|(I-\beta F) z_{m_{k}}-q\right\|^{2} .
\end{aligned}
$$

Combining $1-\mu^{2}>0$ and $\lim _{n \rightarrow \infty} \alpha_{n}=0$, we obtain $\lim _{k \rightarrow \infty}\left\|x_{m_{k}}-y_{m_{k}}\right\|=0$. Using the same argument as in proof of Case 1 , we get $\lim _{k \rightarrow \infty}\left\|x_{m_{k}+1}-x_{m_{k}}\right\|=0$ and

$$
\limsup _{k \rightarrow \infty}\left\langle(I-\beta F) q-q, x_{m_{k}+1}-q\right\rangle \leq 0 .
$$


In fact, since $\lim _{n \rightarrow \infty} \alpha_{m_{k}}=0$, there exists $k_{0} \in \mathbb{N}$ such that $1-2 \alpha_{n} \tau>0$ for all $k>k_{0}$. Therefore, for all $k>k_{0}$, we obtain from (3.19) that

$$
\begin{aligned}
\left\|x_{m_{k}+1}-q\right\|^{2} \leq & \left(1-2 \alpha_{m_{k}} \tau\right)\left\|x_{m_{k}}-q\right\|^{2} \\
& +2 \alpha_{m_{k}} \tau\left[\frac{\alpha_{m_{k}}\left\|x_{m_{k}}-q\right\|^{2}}{2 \tau}+\frac{\left\langle(I-\beta F) q-q, x_{m_{k}+1}-q\right\rangle}{\tau}\right] \\
\leq & \left(1-2 \alpha_{m_{k}} \tau\right)\left\|x_{m_{k}+1}-q\right\|^{2} \\
& +2 \alpha_{m_{k}} \tau\left[\frac{\alpha_{m_{k}} M}{2 \tau}+\frac{\left\langle(I-\beta F) q-q, x_{m_{k}+1}-q\right\rangle}{\tau}\right] .
\end{aligned}
$$

for $M>0$. It implies that

$$
\left\|x_{m_{k}+1}-q\right\|^{2} \leq \frac{\alpha_{m_{k}} M}{2 \tau}+\frac{\left\langle(I-\beta F) q-q, x_{m_{k}+1}-q\right\rangle}{\tau} .
$$

Since $\left\|x_{k}-q\right\| \leq\left\|x_{m_{k}+1}-q\right\|$, we have $\limsup _{k \rightarrow \infty}\left\|x_{k}-q\right\|=0$. This implies the sequence $\left\{x_{k}\right\}$ converges strongly to $q$. From the above, we can conclude that the sequence generated by Algorithm 3.5 converges strongly a point $q$, which is a unique solution of the hierarchical variational inequality

$$
\langle F q, z-q\rangle \geq 0, \quad \forall z \in V I(C, A) .
$$

The proof is completed.

\section{NUMERICAL EXPERIMENTS}

In this section, we give some numerical examples to illustrate the main result of Algorithm 3.1 and Algorithm 3.5. In numerical examples, we choose $\gamma=1, l=\mu=0.5, \alpha_{n}=\frac{1}{n+1}, F(x)=\frac{4}{3} x$. The projection over $C$ is computed effectively by the function quadprog in Matlab 7.0 Optimization Toolbox for Example 4.1.

In the following, we give the specific examples.

Example 4.1. Let we consider the Kojima-Shindo nonlinear complementarity problem [20] with $n=4$ for Algorithm 3. In the following, the feasible set $C$ is defined by

$$
C=\left\{x \in \mathfrak{R}_{4}^{+}: x_{1}+x_{2}+x_{3}+x_{4}=4\right\} .
$$

The operator $A$ is defined by

$$
A\left(x_{1}, x_{2}, x_{3}, x_{4}\right)=\left(\begin{array}{c}
3 x_{1}^{2}+2 x_{1} x_{2}+2 x_{2}^{2}+x_{3}+3 x_{4}-6 \\
2 x_{1}^{2}+x_{1}+x_{2}^{2}+10 x_{3}+2 x_{4}-2 \\
3 x_{1}^{2}+x_{1} x_{2}+2 x_{2}^{2}+2 x_{3}+9 x_{4}-9 \\
x_{1}^{2}+3 x_{2}^{2}+2 x_{3}+3 x_{4}-3
\end{array}\right) .
$$

In this example, we choose different starting points $x_{0}=(1,1,1,1), x_{0}=(2,0,1,1)$ and $x_{0}=(2,0,0,2)$. We use the condition $\left\|x_{n+1}-x_{n}\right\| \leq \varepsilon$ to terminate the Algorithm 3.5. Then we analyze the iterative steps and times of the Algorithm 3.5 from different values of $\varepsilon=10^{-3}, \varepsilon=10^{-4}, \varepsilon=10^{-5}$. The results are shown in Table 1. From the above verification, we can know that our algorithm is effective.

Table 1: Numerical results as regards Example 4.1 


\begin{tabular}{c|c|c|c}
\hline$x_{0}$ & $\varepsilon$ & Iter. Step & Iter. Time \\
\hline$(1,1,1,1)$ & $10^{-3}$ & 183 & 24.14 \\
& $10^{-4}$ & 185 & 25.48 \\
\hline$(2,0,1,1)$ & $10^{-3}$ & 165 & 21.26 \\
& $10^{-4}$ & 174 & 23.95 \\
\hline$(2,0,0,2)$ & $10^{-3}$ & 284 & 55.34 \\
& $10^{-4}$ & 810 & 109.59 \\
\hline
\end{tabular}

Example 4.2. Consider the Algorithm 3.1 in the dimensional Hilbert space $H=L^{2}([0,1])$ with the inner product

and the induced norm

$$
\langle x, y\rangle:=\int_{0}^{1} x(t) y(t) d t, \quad \forall x, y \in H,
$$

$$
\|x\|:=\left(\int_{0}^{1}|x(t)|^{2} d t\right)^{\frac{1}{2}}, \quad \forall x \in H .
$$

Let the operator $A: H \rightarrow H$ be defined by

$$
(A x)(t)=\max \{0, x(t)\}, \quad \forall x \in H .
$$

It is easy to verify that the operator $A: H \rightarrow H$ is monotone and 1-Lipschitz continuous. Let $C:=\{x \in$ $H:\|x\| \leq 1\}$. The solution of the problem is $x^{*}=0$. For starting points $x_{0}(t)=t^{2}$ and $x_{0}(t)=t+0.5 \cos t$, respectively, the numerical results are shown in Table 2 . We use the condition $\left\|x_{n+1}-x_{n}\right\| \leq \varepsilon$ to terminate the Algorithm 3.1. We mainly consider the Algorithm 3.1's iteration step and iteration time (in second) to verify its effectiveness.

Table 2: Numerical results as regards Example 4.2

\begin{tabular}{c|c|c|c}
\hline$x_{0}$ & $\varepsilon$ & Iter. Step & Iter. Time \\
\hline$t^{2}$ & $10^{-1}$ & 7 & 14.76 \\
\hline$t+0.5 \cos t$ & $\frac{1}{2} \cdot 10-1$ & 5 & 7.48 \\
\hline
\end{tabular}

\section{CONCLUSION}

The classical variational inequality problem is an important problem in mathematical research and it plays an important role in nonlinear analysis and optimization problems. Nowadays, there are many different ways to solve the variational inequality problem. In this paper, we studied the weak convergence and strong convergence of a self-adaptive Armijo-like step size method for solving the variational inequality problems with monotone and Lipschitz continuous mapping in a real Hilbert space. They were proposed on the basis of the Tseng's extragradient method. We combined Tseng's extragradient method with a relaxation algorithm and the Yamada's algorithm, respectively. In particular, we proved a strong convergence theorem for finding a point which is a unique of the hierarchical variational inequality without any requirement of additional projections. Some numerical experiments are performed to verify the effectiveness of the Algorithm 3.1 and Algorithm 3.5.

\section{Funding}


This work was supported by Tianjin Key Lab for Advanced Signal Processing, Civil Aviation University of China (No. 2019ASP-TJ02).

\section{REFERENCES}

[1] L.C. Ceng, Q.H. Ansari, J.C. Yao Relaxed extragradient methods for finding minimum-norm solutions of the split feasibility problem, Nonlinear Anal. 75 (2012), 2116-2125.

[2] T.M. Tuyen, A cyclic iterative method for solving a class of variational inequalities in Hilbert spaces, Optimization, 67 (2018), 1769-1796.

[3] X. Qin, A. Petrusel, J.C. Yao, CQ iterative algorithms for fixed points of nonexpansive mappings and split feasibility problems in Hilbert spaces, J. Nonlinear Convex Anal. 19 (2018), 157-165.

[4] N. Nadezhkina, W. Takahashi, Weak convergence theorem by an extragradient method for nonexpansive mappings and monotone mappings, J. Optim. Theory Appl. 128 (2006), 191-201.

[5] F. Mainge, A hybrid extragradient-viscosity method for monotone operators and fixed point problems, SIAM J. Control Optim. 47 (2008), 1499-1515.

[6] A. Gibali, Y. Shehu, An efficient iterative method for finding common fixed point and variational inequalities in Hilbert spaces, Optimization, 68 (2019), 13-32.

[7] D.V. Thong, D.V. Hieu, Inertial subgradient extragradient algorithms with line-search process for solving variational inequality problems and fixed point problems, Numer Algor. 80 (2019), 1283-1307.

[8] G.M. Koepelevich, The extragradient method for finding saddle points and other problem, Ekon. Mat. Metody 12 (1976), 747-756.

[9] P. Tseng, A modified forward-backward splitting method for maximal monotone mappings, SIAM J. Control Optim. 38 (2000), 431-446.

[10] Y. Censor, A. Gibali, S. Reich, The subgradient extragradient method for solving variational inequalities in Hilbert space, J. Optim. Theory Appl. 148 (2011), 318-335.

[11] Q.L. Dong, Y.J. Cho, L. Zhong, Th. M. Rassias, Inertial projection and contraction algorithms for variational inequalities, J. Global Optim. 78 (2018), 687-704.

[12] D.V. Thong, D.V. Hieu, Weak and strong convergence theorems for variational inequality problems, Numer. Algor. 78 (2018), 1045-1060.

[13] I. Yamada, The hybrid steepest descent method for the variational inequality problem over the intersection of fixed point sets of nonexpansive mappings, In: Inherently parallel algorithms in feasibility and optimization and there applications, (2001), 473-504.

[14] K. Goebel, S. Reich, Uniform Convexity, Hyperbolic Geometry, and Nonexpansive Mappings, Marcel Dekker, Nwe York, (1984).

[15] W. Takahashi, Introduction to Nonlinear and Convex Analysis, Yokohoma, Yokohoma Publishers(2009).

[16] R.W. Cottle, J.C. Yao, Pseudo-monotone complementarity problems in Hilbert space, J. Optim. Theory Appl. 75 (1992), 281-295.

[17] H.K. Xu, Iterative algorithm for nonlinear operators, J. London Math. Soc. 66 (2002), 240-256.

[18] H.K. Xu, Averaged mappings and the gradient-projection algorithm, J. Optim. Theory Appl. 150 (2011), 360-378.

[19] P.E. Mainge, The viscosity approximation process for quasi-nonexpansive mapping in Hilbert space, Comput. Math. Appl. 59 (2010), 74-79.

[20] J.S. Pang, S.A. Gabriel, A robust algorithm for the nonlinear complementarity problem, Math. Program. 60 (1993), $295-$ 337. 